\title{
Crystallization Behavior of $\mathrm{HfO}_{2}$ Nanotubes in Different Environments
}

\author{
Parag Banerjee ${ }^{1,2}$, Wen-An Chiou ${ }^{1,3}$ and Gary W. Rubloff ${ }^{1,2,3}$
}

1. Department of Materials Sci. and Engineering, University of Maryland, College Park, MD 20742

2. Institute for Systems Research, University of Maryland, College Park, MD 20742

3. NISP Lab, NanoCenter, University of Maryland, College Park, MD 20742-2831

Development of nanostructures and control of their physical properties is imperative for studying and exploiting a unique array of material characteristics in the nanoscale regime. $\mathrm{HfO}_{2}$ is an important technological material [1], and has been used as a high-k material in semiconductor devices. However, the microstructure of $\mathrm{HfO}_{2}$ is poorly understood, especially when a thin film is placed inside 3-D nanostructures. This paper presents a TEM characterization of the crystallization behavior of amorphous $\mathrm{HfO}_{2}\left(\mathrm{a}-\mathrm{HfO}_{2}\right)$ films under various constrained and unconstrained environments.

A 3-D anodic aluminum oxide (AAO) nanoporous template (Fig. 1a) was utilized to deposit $\mathrm{HfO}_{2}$ films using Atomic Layer Deposition (ALD) technique. The temperature of deposition was kept at $250{ }^{\circ} \mathrm{C}$ to ensure an amorphous $\mathrm{HfO}_{2}$ film was deposited (Fig. 1b). To understand the effect of environmental change on the crystallization behavior of $\mathrm{HfO}_{2}$, two sets of samples were prepared. One set (A) of the film was left inside the AAO 3-D porous network (constrained, Fig. 1c), the second set (B) of nanotubes (NTs) was released by dissolving the template (unconstrained, Fig. 1d). Both sets of NTs were then thermal annealed at $650{ }^{\circ} \mathrm{C}$ for $30 \mathrm{mins}$ in high purity Argon gas. Individual NTs were collected by gentle ultrasonification in alcohol solution, and a small drop of suspension was pipetted onto a Cu grid for TEM (JEOL 2100) and SEM (Hitachi SU-70) studies.

TEM investigation showed that NTs by both techniques were crystallized in thermodynamically stable monoclinic phase (m-HfO $)_{2}$, and only occurred in the thicker regions (Fig. 2, b to d). As the NT wall thickness decreased along its length, smaller crystalline grains were visible (Fig. 2e); until at the very end of the NT, the walls were purely amorphous (Fig. 2f). Assuming the transformation of amorphous to crystalline $(\mathrm{a} \rightarrow \mathrm{c})$ occurs via a nucleation and growth mechanism, a critical nuclei size is required for grain growth. This implies that for regions of the NT where the wall thickness is smaller than the critical nuclei size, no stable nuclei can be germinated and hence $\mathrm{a} \rightarrow \mathrm{c}$ does not occur and the film remains amorphous. Finite sized nuclei have also been observed in $2 \mathrm{~nm}$ thick planar $\mathrm{HfO}_{2}$ films [2]. Figure 3a revealed that the final outer diameter of $\mathrm{B}$ is $\sim 47 \mathrm{~nm}$, much smaller than A or unannealed NTs both of which measure $\sim 70 \mathrm{~nm}$. Significant volume reduction occurred in the free standing NTs as it annealed. To fit the same amount of atoms in a smaller diameter (and hence volume) of the B NTs, crystals must be stressed, twisted and eventually deformed. The high density of defects (dislocations) (Fig. 3b) was caused by this volume reduction. As the wall thickness of NTs reduced, atoms failed to order especially where the wall thickness was smaller than critical nuclei size and thus NTs remained amorphous in the thinner regions. These results shed light on the fundamental process of atomic rearrangements in the transformation of disordered materials to achieve energetically feasible, long-range ordered structure when subjected to constraints in their nanostructure environment.

[1] G. D. Wilk, et al., J.Applied Physics, 89 (10), (2001) 5243-5275.

[2] F. Bohra, B. Jiang, and J. M. Zuo, Applied Physics Letters, 90 (16) (2007).

[3] Special thanks to I. Perez, E. Robertson and L.-C. Lai for helping with sample preparation and TEM. This work was supported by NSF-MRSEC (DMR 05-20471) and NISP Lab at UMD. 

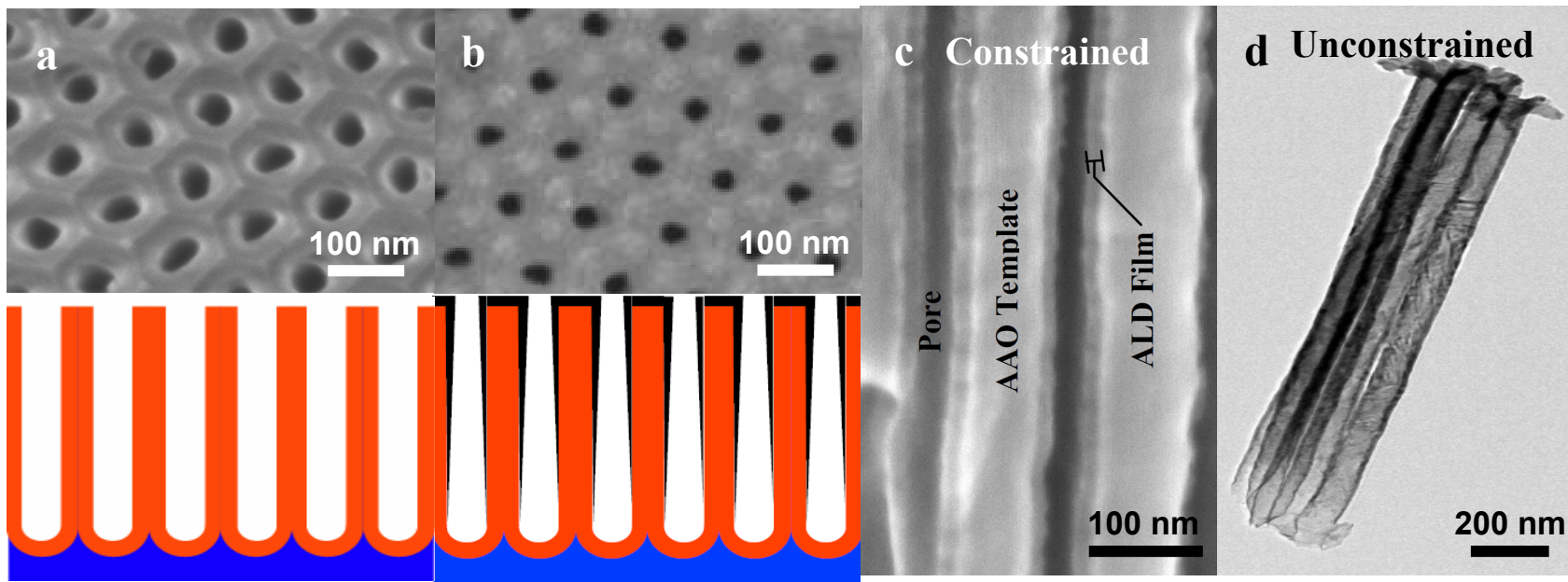

Fig. 1. a-SEM top-down of AAO template and schematic cross section. b- SEM top down after ALD deposition and schematic cross section. $\mathrm{c}-$ SEM cross-sectional view of constrained NT and d-released NT's (TEM).

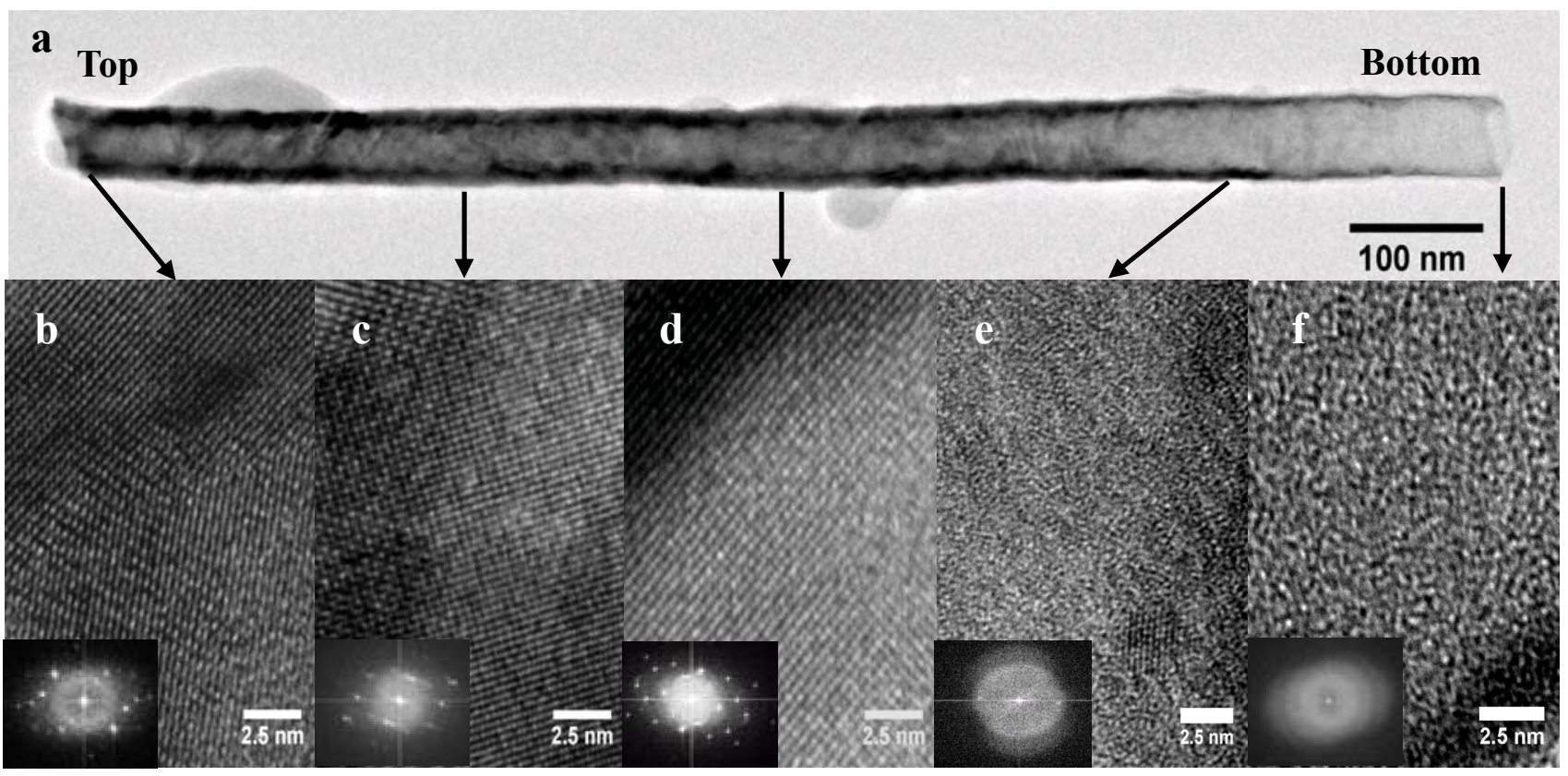

Fig. 2. a- TEM image of a single nanotube. b-f: HRTEM images of different regions along the NT showing a $\rightarrow c$ transformation. Insets show FFT of the HRTEM images. Only $f$ is fully amorphous.

a

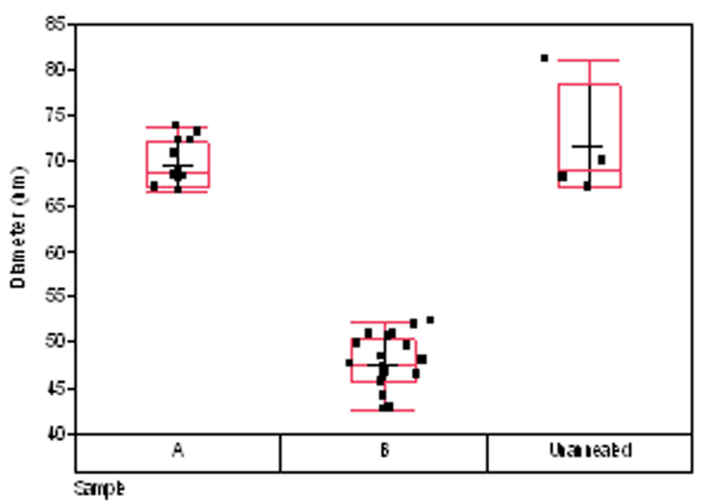

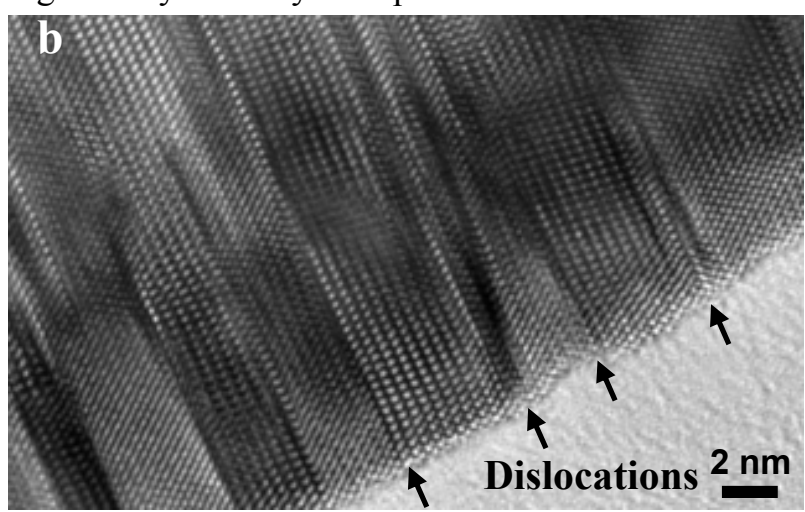

Fig. 3. a - Outer diameters of samples A, B and Unannealed NTs. b - HRTEM of a NT from sample B that depicts defect formation. 